\title{
DESAFIOS DE UM PERIÓDICO CIENTÍFICO JOVEM DE INSTITUIÇÃO PÚBLICA RUMO À INTERNACIONALIZAÇÃO: $O$ caso da Revista Fitos Eletrônica
}

\author{
Rosane de Albuquerque dos Santos Abreu \\ Fundação Oswaldo Cruz, Farmanguinhos \\ rosane.abreu@far.fiocruz.br \\ José Luiz Mazzei \\ Fundação Oswaldo Cruz, Farmanguinhos \\ mazzei@pq.cnpq.br \\ Yolanda de Castro Arruda \\ Fundação Oswaldo Cruz, Farmanguinhos \\ yolanda.arruda@far.fiocruz.br \\ Eugênio Telles \\ Fundação Oswaldo Cruz, Farmanguinhos \\ eugenio.telles@far.fiocruz.br
}

\begin{abstract}
Resumo
O estudo de caso sobre a Revista Fitos Eletrônica, quanto ao processo de internacionalização, revelou o caminho e os desafios pelos quais um periódico jovem de instituição pública enfrenta. Com base na pesquisa de Borini e Ferreira (2015), o estudo apontou que tanto as pressões institucionais quanto a participação em redes de relacionamento internas e externas são os fatores proeminentes que promovem a internacionalização.
\end{abstract}

\section{Palavras-chave}

Periódico científico. Internacionalização. Indexação. Visibilidade. Rede de relacionamentos.

\section{INTRODUÇÃO}

No XV Encontro Nacional de Editores Científicos em 2015, evento patrocinado pela Associação Brasileira de Editores Científicos (ABEC Brasil), a equipe da Revista Fitos Eletrônica (RFE) apresentou, em pôster um estudo de caso sobre os desafios na mudança do processo de editoração de impresso para o eletrônico (ABREU et al, 2015). Após o estabelecimento do periódico na versão online, em 2014, a meta principal foi a de cumprir todo o processo editorial, da submissão à publicação, pelo Sistema Eletrônico de Editoração de Revistas (SEER), versão traduzida e personalizada pelo Instituto Brasileiro de Informação em Ciência e Tecnologia (Ibict) do Open Journal Systems (OJS). No que se refere à estrutura editorial em si, seguiu-se àquela estabelecida para o periódico em modalidade impressa e tentou-se adequar ao sistema eletrônico.

Meadows (2001, p. 1) indica que este movimento não foi exclusivo da RFE, mas uma prática natural dos editores que se propõem fazer esta transposição.

Editores e outros profissionais envolvidos no processo de comunicação científica tendem a ver a publicação em forma eletrônica simplesmente como publicação impressa transferida para um novo meio eletrônico para tratamento e disseminação.

Nesses últimos tempos, profundas mudanças na comunicação e na produção científica brasileira foram identificadas, 
como: crescimento quantitativo dos periódicos brasileiros online (BORINI; FERREIRA, 2015), emergência do Movimento de Acesso Aberto, proliferação de repositórios institucionais e temáticos, entre outros. No que se refere especificamente à comunicação científica, o contexto editorial caracteriza-se pela competitividade, com influência dos grandes Publishers, exigindo-se aos periódicos, tornarem-se cada vez mais profissionalizados e internacionalizados.

O presente trabalho tem por objetivo apresentar o caso da RFE, relatando os desafios e as estratégias usadas pela equipe editorial, visando à internacionalização.

\section{INTERNACIONALIZAÇÃO DE PERIÓDICOS CIENTÍFICOS BRASILEIROS}

Nas últimas décadas, estudos revelam um crescimento quantitativo da produção científica brasileira (PACKER, 2011; VALÉRIO, 2005) gerado, principalmente, pelo aumento dos cursos de pós-graduação e pela reestruturação do sistema de avaliação desses cursos pela Coordenação de Aperfeiçoamento de Pessoal de Nível Superior (Capes). Borini e Ferreira (2015) destacam que o principal desafio que envolve a produção científica nacional não está propriamente no volume publicado, mas na veiculação de artigos qualificados. Acrescentam, ainda, que um dos critérios que marca a relevância de um artigo para o meio científico é o grau de sua internacionalização. Tais autores consideram que um periódico está internacionalizado quando este ultrapassa as fronteiras nacionais não só em seu processo de distribuição, mas pela inserção internacional em seu conselho ou comitê editorial e científico.

A internacionalização dos periódicos, no entender desses mesmos autores, pode ser analisada por três direções: a hospedagem de periódicos nacionais em influentes publishers internacionais; a internacionalização auto conduzida, procurando enquadrar as revistas nas bases de dados abertas como Scientific Electronic Library Online (SciElo) e Redalyc; e a obtenção de indexação em bases como a Thomson Reuters (JCR) e Scopus (H-index). A indexação significa, portanto, O reconhecimento dos periódicos (PACKER, 2011) pela comunidade científica, que direciona a sua produção acadêmica para as revistas indexadas, especialmente nas bases citadas acima, buscando dar visibilidade aos seus estudos.

Santos (2012) conceitua indexação como o processo que consiste em recuperar, selecionar e exprimir as informações contidas nos documentos, através de termos pertencentes a uma ou várias linguagens documentais. Diz respeito a uma operação de descrição interna, cujo objeto é o conteúdo intelectual dos documentos e é necessário o cumprimento de várias etapas que facilitam as buscas documentais.

Preparar um periódico para avaliação das agências indexadoras exige atenção às etapas e critérios propostos por estas instituições. Por sua vez, estar indexado em bases cientificamente reconhecidas, especialmente no contexto internacional, é condição básica para os periódicos que visam à internacionalização. Uma etapa decisiva deste processo diz respeito à organização das citações dos artigos em outros periódicos, especialmente os internacionais, com vistas à ampliação da visibilidade, com resultados nos índices de impacto.

Costa apud Borini e Ferreira (2015) afirma que a produção científica brasileira, em 2012, era pouco internacionalizada. Os pesquisadores brasileiros, em sua maioria, têm sido citados prioritariamente em periódicos nacionais e têm dificuldades em publicar seus estudos nos periódicos internacionais.

Em estudo sobre a internacionalização de periódicos brasileiros da área de administração, Borini e Ferreira (2015) consideram dois fatores básicos que norteiam o processo de internacionalização de um periódico, a saber: pressão do ambiente institucional e a inserção em redes de relacionamentos.

No primeiro fator, os autores identificam a influência da Capes, exercendo pressões e impulsionando a 
internacionalização dos periódicos nacionais com a exigência de indexação em bases internacionais reconhecidas (Scopus e Thompson Reuters), o que amplia o fator de impacto dos periódicos. Outra pressão da Capes é a padronização dos periódicos, pois se considera como modelo de excelência as revistas classificadas nos estratos mais altos, assim estes se tornam referência para os demais. Outro exemplo de padronização das revistas é a adoção do SEER, software recomendado pela Capes, desenvolvido para gerenciamento e publicação de revistas eletrônicas, que permite uma melhoria na avaliação da qualidade dos periódicos e maior rapidez no fluxo das informações. A segunda categoria proposta por Borini e Ferreira (2015), refere-se à inserção do periódico em redes de relacionamentos. Ressalta-se o papel estratégico dos editores, como interlocutores com o corpo editorial e consultores ad hoc, para atrair membros internacionais e de renome em sua área de atuação para fazer parte da rede.

\section{INTERNACIONALIZAÇÃO QUALIDADE DE CONTEÚDO}

Trzesniak, Plata-Caviedes e CórdobaSlagado (2012) sinalizam um único desafio para as duas próximas décadas, que impactará todos os segmentos e atores envolvidos na construção e divulgação do conhecimento que é a qualidade de conteúdo.

No contexto da comunicação científica, portanto, cuidar-se da qualidade do conteúdo veiculado no periódico é condição básica para se buscar a confiabilidade e respeitabilidade da comunidade científica e, consequentemente, impactar o processo de internacionalização de um periódico.

As mudanças trazidas pelas Tecnologias de Informação e Comunicação (TICs) influenciaram fortemente o fazer ciência, a comunicação científica e trouxeram a quebra da ideia de que um artigo publicado havia passado por criterioso processo editorial e, consequentemente, apresentava qualidade de conteúdo assegurada. Estes mesmos autores alertam que os tempos mudaram e não é mais verdadeiro o pressuposto de que o que foi processado editorialmente e publicado tem qualidade.

$\mathrm{O}$ excesso de informação e conhecimentos, a rapidez com que estes circulam, os variados recursos para cópia e recuperação de dados, contribuíram para a quebra desse pressuposto. Por outro lado, o paradigma da quantificação que tomou conta da área científica, tanto na avaliação de pesquisadores, quanto na dos periódicos, produziram fenômenos que impactaram a qualidade do conhecimento científico produzido e publicado.

Para a recuperação da qualidade, os autores orientam que é preciso atentar, equilibradamente, para o cuidado com três "logias", a saber: terminologia, cuidando do rigor e do compartilhamento dos conceitos; epistemologia, promovendo o avanço do conhecimento e a metodologia, que diz respeito à maneira correta e rigorosa de fazer pesquisa.

Para a melhoria da qualidade, os autores reafirmam o importante papel dos editores científicos, que devem manter uma atuação didática sobre a comunidade, auxiliando autores e revisores a identificar todos e cada um dos requisitos que um artigo deve atender para atingir a qualidade plena.

\section{SITUAÇÃO DE INDEXAÇÃO DE PERIÓDICOS RELACIONADOS AO ESCOPO DA RFE}

A RFE é um periódico interdisciplinar, em acesso aberto, de difusão gratuita, com periodicidade trimestral e publica artigos científicos originais sobre Pesquisa, Desenvolvimento e Inovação (PD\&I) em medicamentos da diversidade vegetal. Em pesquisa realizada em algumas bases de dados internacionais, verificou-se que os periódicos científicos brasileiros correntes, listados na base Ulrichsweb (2017) e com grande número de publicações de artigos diretamente relacionados ao escopo da RFE, com foco em Plantas Medicinais, encontramse classificados pela Capes em B2 (16\%) a C na área de Farmácia, sendo a maioria em B3 $(50 \%)$. Não diferentemente de periódicos em outras áreas, são reconhecidos com maior 
qualificação na área interdisciplinar, principalmente em 1 a 2 níveis, embora nenhum periódico da área tenha qualificação acima de B1. Este panorama está ilustrado na Figura 1. Uma característica importante é que $60 \%$ são de acesso aberto, dentre estes apenas
2/3 passam por revisão de pares no processo editorial e apenas dois periódicos não são mais publicados em versão eletrônica (ULRICHSWEB, 2017), como representado na Figura 2.

Figura 1. Número de periódicos nacionais com alta frequência de artigos em plantas medicinais nas classificações Qualis Periódicos/Capes

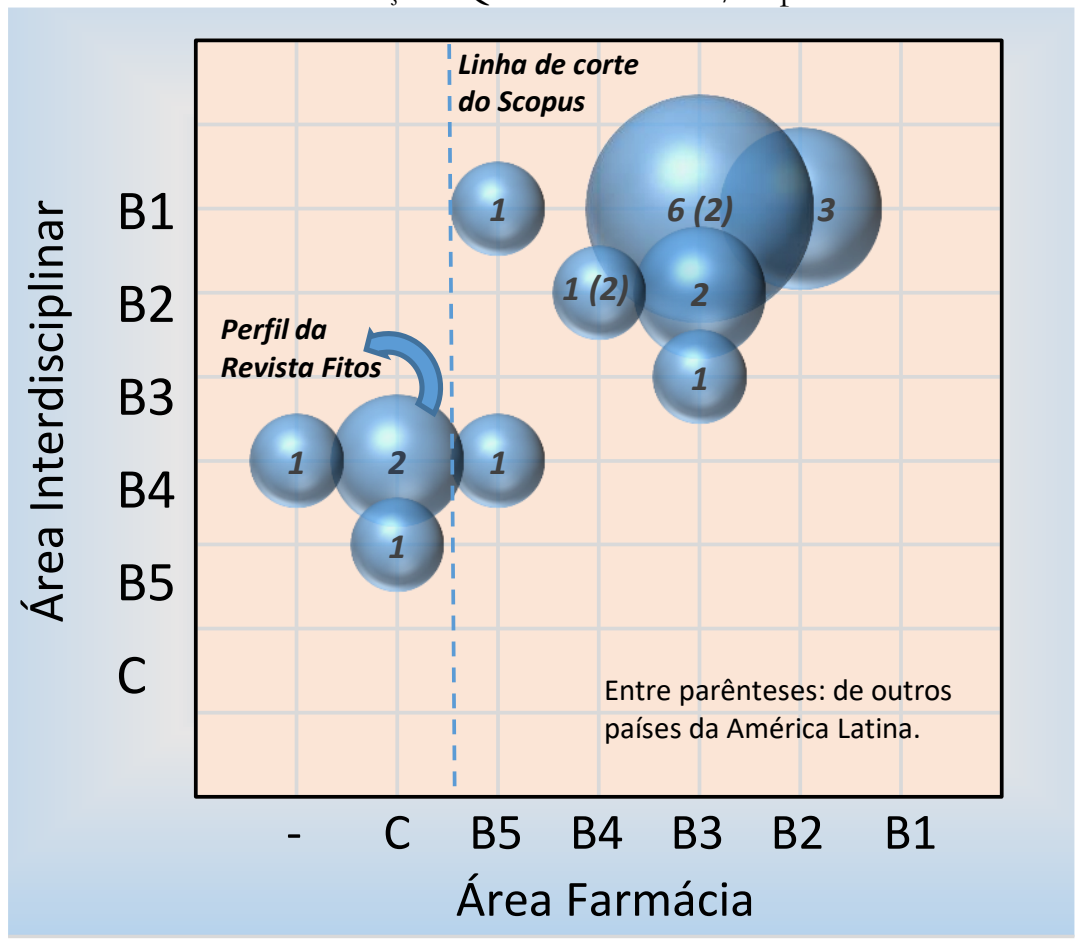

Fonte: Ulrichsweb (2017); Capes (2017).

Figura 2 - Características de acesso e do processo editorial de periódicos nacionais com alta frequência de artigos em plantas medicinais

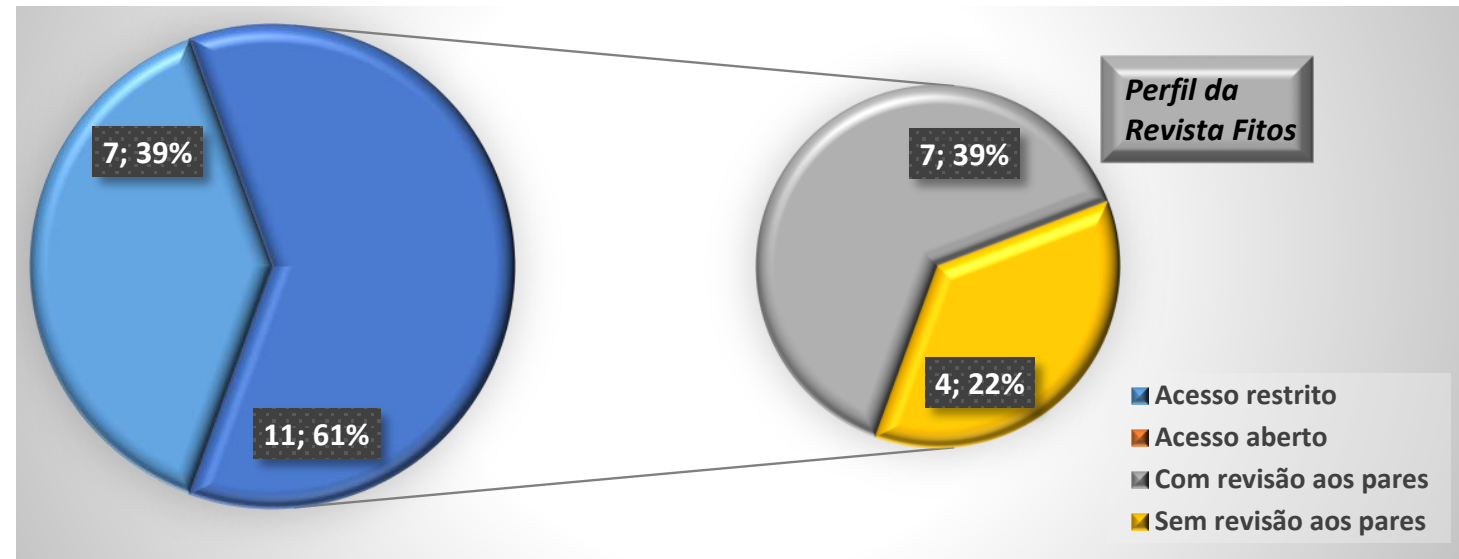

Fonte: Ulrichsweb (2017).

Este reconhecimento, de razoável a fraco, não é tratado diferentemente nos poucos periódicos renomados da área e publicados em demais países da América
Latina, principalmente no Chile, Costa Rica e Cuba. À exceção de apenas quatro brasileiros, os periódicos da América Latina estão indexados na base Scopus (2017), enquanto a 
Principal Coleção da Web of Science (2017), assim como na base SciElo (2017), apresenta metadados de cerca da metade dos periódicos da área. Afora única exceção, os periódicos da América Latina que não estão indexados na Scopus são os únicos em classificação $C$ pela Capes na área de Farmácia.

Este panorama, representado na Figura 3, demonstra o quanto a RFE ainda precisa caminhar, assim como evidencia os desafios a serem enfrentados para conquistar uma qualificação de reconhecimento internacional, mesmo tendo características pertinentes de versão eletrônica de acesso aberto, com revisão pelos pares no processo editorial. $\mathrm{O}$ esforço para conquistar a indexação com a Scopus é, certamente, a melhor opção de passo inicial.

Figura 3. Coleções nas principais bases de metadados de periódicos da América Latina com alta frequência de publicações em plantas medicinais

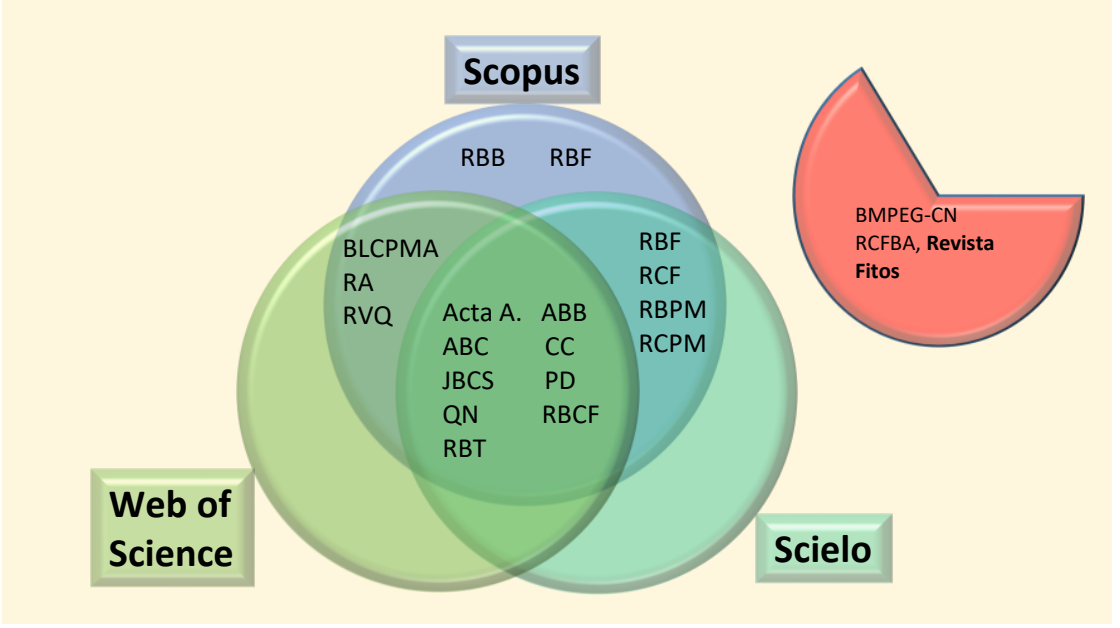

Fonte: Ulrichsweb (2017).

Isto posto, para um periódico científico chegar à internacionalização, tem que ter a percepção das pressões institucionais e estar fortemente inserido em rede externa de relacionamentos (BORINI; FERREIRA, 2015). Assim, a internacionalização não se dá de forma rápida, mas em várias etapas. Para tanto, a equipe editorial da RFE apresenta abaixo um estudo de caso, que destaca as ações e estratégias tomadas visando a internacionalização.

\section{METODOLOGIA}

Para apresentar o estudo de caso foi realizada pesquisa documental, com buscas e análise de documentos editoriais, memórias de reunião, relatórios, projetos, além de observações registradas no próprio processo de trabalho. O material foi lido e analisado, e seus resultados tomaram por base os dois fatores propostos por Borini e Ferreira (2015).

\section{O CASO DA REVISTA FITOS ELETRÔNICA}

A RFE é editada em formato eletrônico, desde 2014, pelo Núcleo de Gestão em Biodiversidade e Saúde (NGBS) do Instituto de Tecnologia em Fármacos/Farmanguinhos/Fiocruz.

O NGBS tem por missão a promoção da inovação em medicamentos da biodiversidade, desenvolvida através de um Sistema Nacional de Redes - RedesFito, organizada a partir de arranjos produtivos locais, identificados nos seis biomas nacionais (REDESFITO, 2017). Baseia-se na concepção da inovação como um processo dinâmico e social que se dá por ações colaborativas de diferentes atores integrantes da cadeia de desenvolvimento de um medicamento da biodiversidade. Nesse contexto, o processo de produção e circulação de conhecimentos, e aqui destaca- 
se o conhecimento científico, tem o papel central para que a inovação ocorra. Assim, considera-se estratégico para o NGBS a edição de um periódico científico proposto para ser o veículo de comunicação científica para as RedesFito, além do desenvolvimento de um curso de pós-graduação lato sensu, em modalidades presencial e à distância, realizado em articulação com o setor de ensino da unidade, entre outros serviços.

Há que se destacar que Farmanguinhos é uma unidade da Fiocruz responsável pela produção de medicamentos, que tem por missão a promoção da saúde pública por meio da geração e difusão de conhecimentos, do ensino, da pesquisa, do desenvolvimento tecnológico e da produção de medicamentos. $\mathrm{Na}$ área de ensino, Farmanguinhos promove um curso de mestrado profissional em Gestão, Pesquisa e Desenvolvimento na Indústria Farmacêutica e dois cursos de especialização lato sensu, sendo um deles o do NGBS citado acima.

A RFE recebe apoio financeiro, exclusivamente, de Farmanguinhos e sofre, portanto, as consequências geradas pelas crises no setor público.

\section{OS DESAFIOS E ESTRATÉGIAS USADAS PELA EQUIPE EDITORIAL}

Como foi dito, o desafio inicial da RFE em 2015 foi o de implantar definitivamente o processo editorial através do SEER, sem fazer alterações na política e na estrutura editorial. Atualmente, novos desafios emergem, visando à internacionalização, a saber: obter indexação em novas bases de dados nacionais e internacionais; aumentar a visibilidade; melhorar a qualidade dos manuscritos e ampliar a rede de relacionamentos com pesquisadores e instituições nacionais e internacionais. A seguir, serão apresentadas as estratégias usadas pela equipe editorial, com vistas à internacionalização.

\subsection{Adequação do foco e escopo}

Anteriormente, o foco e escopo não colocavam em destaque PD\&I em medicamentos da biodiversidade e deixava transparecer que a RFE era mais uma publicação sobre plantas medicinais. Além disso, restringia-se aos biomas brasileiros, deixando de fora os demais biomas da Terra, fundamentais para a internacionalização. A maior clareza no foco e a retirada da restrição foram propostas pela equipe editorial $\mathrm{e}$ atualmente são fundamentais para a captação dos trabalhos internacionais.

Um estudo realizado por alguns editores sobre as áreas do conhecimento reconhecidas pelo Conselho Nacional de Desenvolvimento Científico e Tecnológico (CNPq) e da Capes, com interface ao foco e escopo da RFE, revelou a necessidade de ampliação de áreas para a recepção de manuscritos. Assim, foram incorporadas quatro novas áreas, reafirmando o caráter interdisciplinar da RFE e contemplando-se trabalhos em áreas afins.

Ampliou-se, então, a vocação da revista, sem perder o foco de atuação e mantendo o rigor qualitativo com o convite a pesquisadores dessas novas áreas para atuar na editoração específica.

\subsection{Ampliação da visibilidade}

Para facilitar a localização rápida de autores e artigos na rede, exige-se uma identificação de autor (ORCID, ResearchId, etc), assim como todos os artigos publicados pela RFE recebem a identificação do Digital Object Identifier System (DOI), da associação CrossRef.

A apresentação correta das citações e referências torna-se essencial. A RFE informa a identificação International Standard Serial Number (ISSN) ou International Standard Book Number (ISBN) (periódicos e livros) de todas as referências citadas em seus artigos, bem como os hiperlinks do DOI, PubMed e de sites citados e consultados, oferecendo aos autores/leitores o acesso imediato ao documento citado. Procura-se manter todas as referências acessíveis, com a verificação e correção, no momento da revisão final.

Ainda, para a visibilidade, utiliza-se uma fanpage no Facebook que publica sumário e notícias correlatas. O sumário de cada 
edição é enviado por e-mail a uma base de aproximadamente 3000 pessoas cadastradas no Sistema OJS e na RedesFito, procurando divulgar o conhecimento produzido e impactando a rede para a formulação de projetos inovadores em pesquisa e desenvolvimento de medicamentos da biodiversidade.

A participação em eventos científicos para divulgação da revista também é uma estratégia utilizada, com vistas à ampliação da visibilidade. De fato, em 2017 a equipe editorial promoveu a RFE em diversos eventos científicos, tanto em áreas de pesquisa em plantas e produtos naturais, quanto em editoria científica.

Há ainda um folder eletrônico usado por diferentes agentes para captação de artigos. Desenvolve-se um trabalho sistemático de divulgação do periódico junto à grupos de pesquisa e em coordenações de pós-graduação das áreas afins, promovendo a visibilidade da revista em diferentes espaços.

\subsection{Promoção da rede de relacionamentos}

O papel estratégico dos editores na internacionalização de periódicos é importante como uma cadeia de relacionamento direcionados para captação de artigos de qualidade. Avaliando a atuação de cada editor, com critérios definidos, foi concluída a necessidade de renovação do Corpo Editorial e dos Editores de Áreas, o que ocorreu 2017.

Hoje, a coordenação científica da revista é constituída por três editores pesquisadores de Farmanguinhos, contando com a colaboração de 12 editores das áreas de submissão. A escolha destes editores foi definida por critérios de reconhecimento acadêmico e da vinculação aos programas de pós-graduação de universidades e instituições diversas. Assim, formou-se uma rede de editores distribuída por vários estados e instituições de ensino e pesquisa do Brasil. A interlocução entre esses editores ocorre não apenas para as questões científicas, mas para as questões editoriais mais amplas. Para tanto, a editoria executiva mantem um comunicado mensal, em que são informadas as demandas, as dificuldades e as estratégias usadas para a superação destas e realiza reuniões virtuais, sempre que necessário.

$\mathrm{Na}$ estratégia de interação em rede, a participação efetiva da equipe editorial da RFE no Fórum de Editores da Fiocruz reforça a importância da interação entre os editores das demais revistas da instituição. Há a preocupação, também, em alimentar com notícias e informações o Portal de Periódicos da Fiocruz.

\subsection{Melhoria da qualidade dos manuscritos}

Visando a internacionalização da RFE, os critérios para submissão e avaliação dos manuscritos tornaram-se mais exigentes, mesmo tendo baixa quantidade de submissões. Optou-se por construir confiabilidade e respeitabilidade científica, mesmo que isso custasse indexação.

Outra estratégia usada foi a ampliação a três idiomas do texto principal, aceitando-se manuscritos, além do português, em inglês e espanhol.

Houve, também, mudança da norma da ABNT para Vancouver, o que gerou reformulação nas instruções aos autores.

A RFE, zelando pela publicação de qualidade e em consonância com as demais revistas da Fiocruz, segue as orientações do Committee on Publication Ethics (COPE).

\section{CONSIDERAÇÕES FINAIS}

Face ao que foi exposto, fica explícito o objetivo da equipe editorial da RFE de levar o periódico à internacionalização. As decisões editoriais vieram a corroborar o estudo de Borini e Ferreira (2015), revelando que as estratégias implementadas na RFE levaram em conta a pressão do ambiente institucional, assim como a preocupação com a construção da rede de relacionamentos. Estes mesmos autores concluíram que os periódicos mais propensos à internacionalização são aqueles que trabalham segundo essas categorias. Muitos passos ainda precisam ser dados, mas fica a percepção de que o caminho trilhado 
pela equipe editorial da RFE tem sido apropriado.

\title{
CHALLENGES TOWARDS INTERNATIONALIZATION OF A YOUNG SCIENTIFIC JOURNAL OF PUBLIC INSTITUTION: THE CASE OF REVISTA FITOS ELETRÔNICA
}

\begin{abstract}
The case study on the journal Revista Fitos Eletrônica, regarding the process of internationalization, revealed the path and the challenges that a young scientific journal of public institution faces. Based on the research by Borini and Ferreira (2015), the study pointed out that both institutional pressures and participation in internal and external network of relationships are the prominent factors that promote internationalization.
\end{abstract}

Keywords

Scientific periodical journal. Internationalization. Indexing. Visibility. Network of relationships. Artigo recebido em 15/01/2018 e aceito para publicação em 18/02/2018

\section{REFERÊNCIAS}

ABREU, R. A. S.; TELLES, E. F.; ARRUDA, Y. C.; DA SILVA, D. M.; DE OLIVEIRA, P. J. M.; PONTES, T. V. C. Desafios na transição de periódico impresso para eletrônico: o caso da Revista Fitos. In: ENCONTRO NACIONAL DE EDITORES CIENTÍFICOS, 15., 2015, Florianópolis. Anais... Florianópolis: Associação Brasileira de Editores Científicos, 2016. P. 1-5. Disponível em: $<$ https://www.abecbrasil.org.br/eventos/xv enec/index.asp >. Acesso em: 17 maio 2017.

BORINI, Felipe Mendes; FERREIRA, Jackeline. Internacionalização de Periódicos Científicos Brasileiros: Estudo de Caso à luz da Teoria de Redes e da Teoria Institucional. Revista Ibero-Americana de Estratégia, v. 14, n. 4, p. 24, 2015. Disponível em: < http://www.revistaiberoamericana.org/ojs/i ndex.php/ibero/article/view/2252>.

Acesso em: 17 maio 2017.

BRASIL. Coordenação de Aperfeiçoamento de Pessoal de Nível Superior. Classificação de Periódicos Quadriênio 2013-2016. Disponível em: $<$ https://sucupira.capes.gov.br/sucupira/pu blic/consultas/coleta/veiculoPublicacaoQua lis/listaConsultaGeralPeriodicos.jsf $>$.

Acesso em: 02 out. 2017.
Fundação Oswaldo Cruz. Redesfito. Inovação em Medicamentos da Biodiversidade. Disponível em:

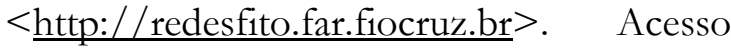
em: 06 out. 2017.

MEADOWS, Arthur Jack. Os periódicos científicos e a transição do meio impresso para o eletrônico. Revista de Biblioteconomia de Brasília, v. 25, n. 1, p. 5-14, 2001. Disponível em: < http://www.brapci.inf.br/index.php/article /view/0000000635/7d52e517c525e6d738ca 3781de134e88 >. Acesso em: 20 set. 2017.

PACKER, Abel L. Os periódicos brasileiros e a comunicação da pesquisa nacional. Revista USP, n. 89, p. 26-61, 2011. Disponível em: < https://www.revistas.usp.br/revusp/article/ view/13868>. Acesso em: 02 out. 2017.

SANTOS, Gildenir Carolino. Fontes de indexação para periódicos científicos: um guia para bibliotecários e editores. EColor, 2012.

TRZESNIAK, Piotr; PLATA-CAVIEDES, Tatiana; CÓRDOBA-SALGADO, Oscar Alejandro. Qualidade de conteúdo, o grande desafio para os editores científicos. Revista Colombiana de Psicologia, v. 21, n. 1, p. 57-78, 2012. Disponível em: $<$ http://www.redalyc.org/articulo.oa?id $=80$ 424036005>. Acesso em: 10 out. 2017. 
VALÉRIO, Palmira Maria Caminha Moriconi. Periódicos científicos eletrônicos e novas perspectivas de comunicação e divulgação para a ciência. 2005. 214 f. Tese (Doutorado em Ciência da Informação) - Universidade Federal do Rio de Janeiro / Instituto Brasileiro de Informação em Ciência e Tecnologia, Rio de Janeiro, 2005. Disponível em: $<$ http://ridi.ibict.br/handle/123456789/68 7>. Acesso em: 16 set. 2017.

SCIENTIFIC ELECTRONIC LIBRARY ONLINE. Pesquisa. Disponível em:

i Versão revista e apliada do Trabalho "Desafios de um periódico científico jovem de instituição pública rumo à internacionalização: o caso da Revista Fitos Eletrônica" apresentado no evento ABEC MEETING, 6-9, novembro, Curitiba, PR, 2017.
$<$ http://www.scielo.org/php/index.php $>$. Acesso em: 06 out. 2017.

SCOPUS. Pesquisa. Disponível em: $<$ https://www.scopus.com/search/form.uri ?display=basic $>$. Acesso em: 06 out. 2017.

\section{ULRICHSWEB. Global Serials Solutions.} Disponível em: $<$ https://ulrichsweb.serialssolutions.com/ $>$. Acesso em: 05 out. 2017.

WEB OF SCIENCE. Principal Coleção. Disponível em: < webofknowledge.ez68.periodicos.capes.gov. br>. Acesso em: 06 out. 2017. 